\title{
Object Detection and Tracking using Background Subtraction and Connected Component Labeling
}

\author{
Asad Abdul Malik \\ Institute of Information \& \\ Technology, \\ Kohat University of Science \& \\ Technology, \\ Kohat, Pakistan
}

\author{
Amaad Khalil \\ Department of Computer Systems \\ Engineering \\ University of Engineering \& \\ Technology, \\ Peshawar, Pakistan
}

\author{
Hameed Ullah Khan \\ Department of Computer Systems \\ Engineering \\ University of Engineering \& \\ Technology, \\ Peshawar, Pakistan
}

\begin{abstract}
ABSTRAC
Digital image processing is one of the most researched fields nowadays. The ever increasing need of surveillance systems has further on made this field the point of emphasis. Surveillance systems are used for security reasons, intelligence gathering and many individual needs. Object tracking and detection is one of the main steps in these systems. Different techniques are used for this task and research is vastly done to make this system automated and to make it reliable. In this research subjective quality assessment of object detection and object tracking is discussed in detail. In the proposed system the background subtraction is done from the clean original image by using distortion of color and brightness. The subtracted image is then tracked using connected component labeling. The proposed system eliminates the shadow and provides $79 \%$ accuracy.
\end{abstract}

\section{Keywords}

Object tracking, detection, Background Subtraction, color distortion

\section{INTRODUCTION}

The detection of a moving object and tracking of different objects in a video or video sequence is a very important task in the surveillance videos, analysis and monitoring of traffic, tracking and detection of humans and different gesture recognitions in human-machine interface [1]. The technique of Object tracking can be explained to be the method of tracking the different number of objects in the video and also the certain directions those objects are traversing in and also to track the entrances to the surveillance site as per the unit time. The sophistication and the complexity of the system determine the resolution of the measurement. This system is often deployed in public places such as shopping malls, metro stations, airports and independent surveillance requests. Different approaches can be used for the surveillance and different technologies used as computer vision, infrared beams and thermal imaging [2].

The reasons for object tracking are many For example People counting in retail stores for intelligence gathering can be regarded as one. This is used for the calculation of the conversion rate and rating of the store by the number of customers to the store rather than the old use of the sales data [2].
The video analysis can be safely stated to be consisting of three steps: $1^{\text {st }}$ detecting the objects that are interested in, $2^{\text {nd }}$ the frame to frame tracking of those objects and $3^{\text {rd }}$ the analysis of the path traversed to analyze their behavior [3].

The agenda of this paper is as follows. Section 2 presents Literature that was studied for the background study and better understanding the topic. Section 3 describes the proposed system in view of the study and the current research. Section 4 shows results of the proposed system of this research. Discussion is done is Section 5 and Section 6 contains the conclusion of the current work.

\section{LITERATURE REVIEW}

\subsection{Object Detection}

One of the simplest techniques for the object detection is the background subtraction. In this process the observed frame or image is compared with the same scene but with the exclusion of any objects in the scene. The subtraction from the original scene results in the difference of the two images. The difference highlights the areas with significant change and hence identifies the areas of interest [14,4].

The technique for the background modeling can broadly be classified in two categories i) Non-Recursive and ii) Recursive.

\subsubsection{Non-Recursive Technique}

This technique involves the use of storing the previous $\mathrm{L}$ video frames. The estimation of the background is done with the temporal variation of each pixel within the buffer [5]. A few non-recursive techniques used commonly are described below.

\subsubsection{Frame differencing}

This technique arguably is one of the simplest techniques for background modeling. The video frame used as a background for the frame at time $t$ is the frame at time $t-1$ is used [6].

\subsubsection{Median filtering}

This technique defines the background estimation as a median of all the frames in the buffer at each pixel location processed. It is also assumed that for half of the frames in the buffer the pixel stays in background $[6,15]$. 


\subsubsection{Linear predictive filter}

In this technique the current background is computed by applying linear predictive filter to the pixels of the frames in the buffer. It also estimates the filter coefficients [6].

\subsubsection{Non-parametric models}

This model has the model structure determined from the data and not specified from a priori. So it is different from parametric model. This however does not imply that it does not have parameters but rather the parameters it has are flexible and not fixed [7].

\subsubsection{Recursive Technique}

This technique updates a single background model recursively based on the input frames. So the current model can be significantly affected from the frames of the distant past.

Some of the representative recursive techniques are described below:

\subsubsection{Approximated median Filter}

This technique has the scheme of increasing by one the running estimate of the median if the input pixel is larger than the estimate and so on decremented by one if the input pixel is smaller than the estimate [6].

\subsubsection{Kalman Filter}

It is a widely-used recursive technique for tracking linear dynamical systems under Gaussian noise [3].

\subsubsection{Mixture of Gaussian (MoG)}

This technique uses the method of tracking multiple Gaussian distributions simultaneously $[6,11,12,13]$.

\subsection{Object Tracking}

Object tracking is one of the leading areas of research and quiet important one in the field of computer vision [10]. The availability of higher grade processors, higher quality cameras and the need of automated video analysis has further on spiked the interest in the object tracking algorithms [8].

The main tracking categories of tracking are [9] i)Point Tracking, ii) Kernel Tracking and iii) Silhouette Tracking

\subsubsection{Point Tracking}

In this technique the object is represented by points. These points are detected in consecutive frames to detect the object. These points are associated on the previous knowledge of the points which shows the object motion and positions in previous frames. An external mechanism is required to detect the objects from each frame [9].

\subsubsection{Kernel Tracking}

In the technique the appearance and shape of object is referred to by the kernel. The kernel can be any shape as an elliptical shape or round or a square shape. The motion of the kernel is tracked in various consecutive frames so as to track the motion of the object. This motion of the kernel can be translation, affine or rotational i.e. parametric transformation [9].

\subsubsection{Silhouette Tracking}

In this technique the region of the object in each frame is estimated. This method uses the encoded information inside the region of the object estimated. The encoded information of the object region can be of the form of shape density or appearance density. These are usually of the edge maps form [9].

\section{PROPOSED SYSTEM}

In this system the background subtraction is based on pixel color distortion and brightness distortion are used to identify the candidate foreground.

In this system each pixel of each frame (background frame and upcoming frame) are compared to identify the foreground. When the foreground is identified the shadow elimination technique is applied to remove the shadow of the foreground. And as the background subtracted image is so much sharp. So therefore for reducing that sharpness median filtering is used.

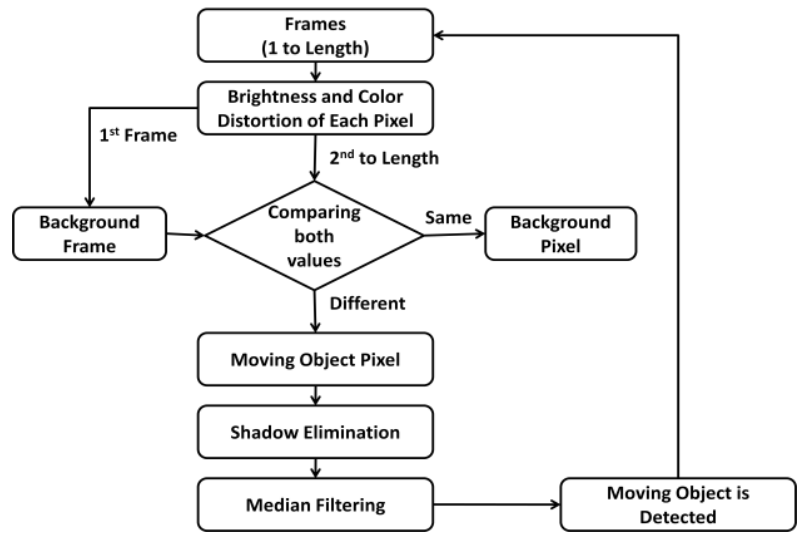

Fig 1: Object Detection Flow Chart

As shown in the flow chart. When the video starts the $1^{\text {st }}$ frame color distortion and brightness distortion is calculated and after that that frame is selected as background model. After selecting the background model, the $2^{\text {nd }}$ frame till the length of frame is upcoming frames. And each frame color and brightness distortion is calculated and compared with the background model. If the values are same then it is considered as background else it is foreground. After finding the foreground shadow elimination technique is used to remove unwanted shadow. After this the median filtering is used for reducing the sharpness of the image. And after all these steps moving object is detected.

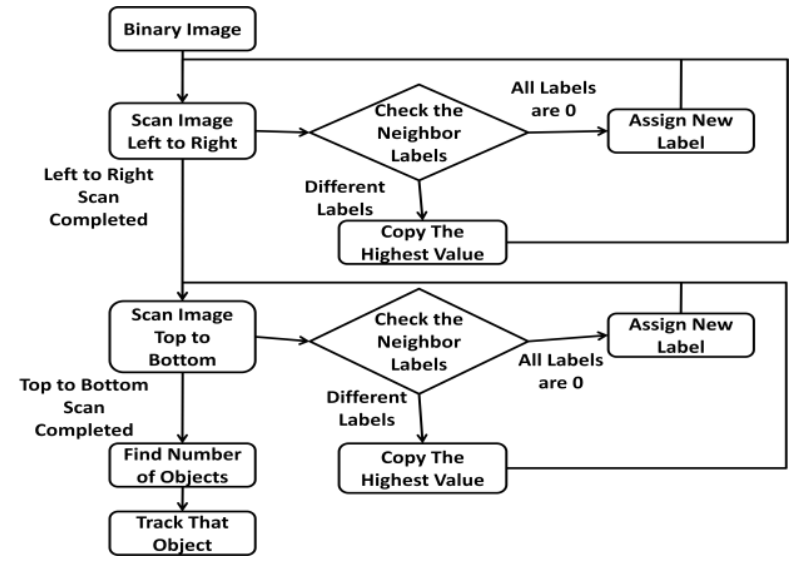

Fig 2: Object Tracking Flow Chart 
For object tracking connected components labeling is used. With the help of this first it finds the boundaries of the object and after that it finds that how many number of objects are present in there video sequence and after that it tracks that object with the help of a rectangular box.

The output of object detection algorithm is selected as input and input image is scanned from left to right and assign labels in such a way that if no neighbors is having a label, it will assign new label. If neighbors have different labels then it will select the highest value of label and assign it to pixel. When the scan from left to right is completed, then scan from top to bottom is performed in a manner performed in previous scan. When both scans are completed, total number of objects is calculated and these objects are tracked.

\section{RESULTS AND DISCUSSION}

In this section subjective quality assessment of proposed algorithm is discussed.

\subsection{Results of Object Detection}

Fig 3(a) is the second frame of test video. 3(b) is the result of subtracting the second frame from the reference or background frame.

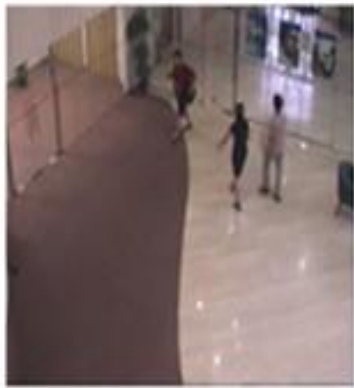

(a)

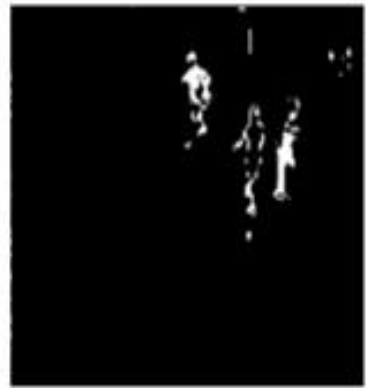

(b)
Fig 3: (a) Second Frame of Video (b) Background Subtraction

Fig 4(a) is the third frame of test video. Fig 4(b) is the result of subtracting the third frame from the reference or background frame.

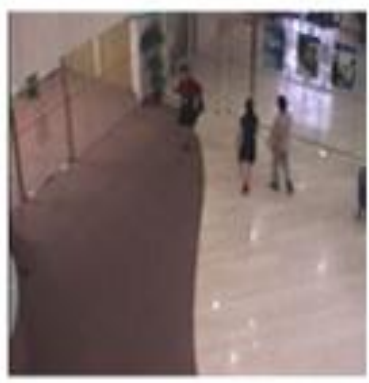

(a)

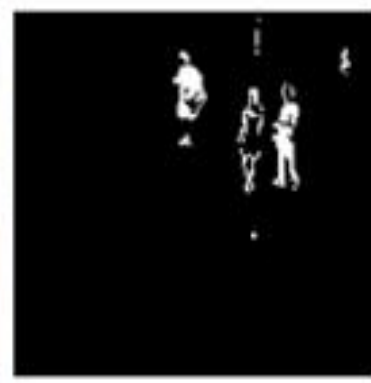

(b)
Fig 4: (a) Third Frame of Video (b) Background Subtraction

Fig 5(a) is the fourth frame of test video. Fig 5(b) is the result of subtracting the fourth frame from the reference or background frame.

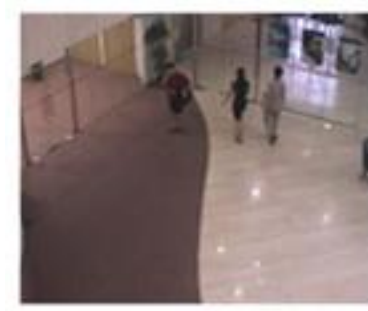

(a)

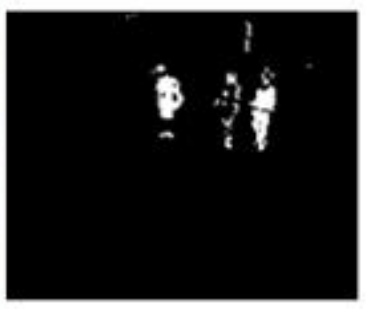

(b)
Fig 5: (a) Fourth Frame of Video (b) Background Subtraction

Fig 6(a) is the fifth frame of test video. Fig 6(b) is the result of subtracting the fifth frame from the reference or background frame.

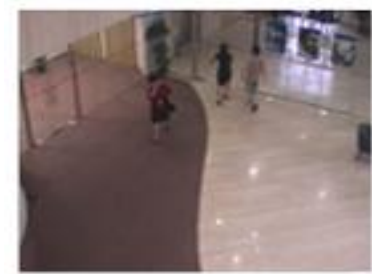

(a)

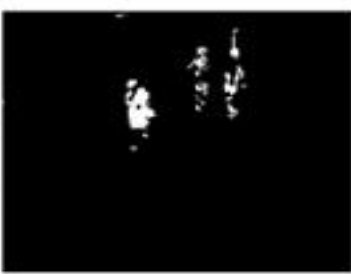

(b)
Fig 6: (a) Fifth Frame of Video (b) Background Subtraction

Fig 7(a) is the sixth frame of test video. Fig 7(b) is the result of subtracting the sixth frame from the reference or background frame.

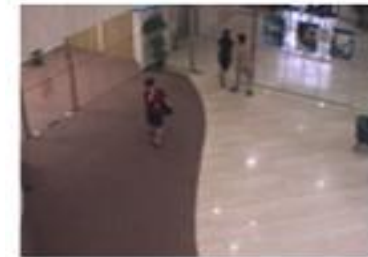

(a)

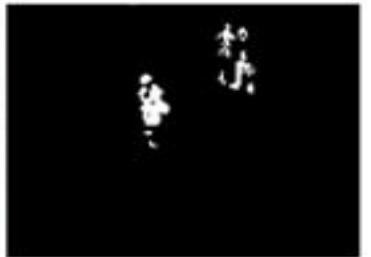

(b)
Fig 7: (a) Sixth Frame of Video (b) Background Subtraction

Fig 8(a) is the seventh frame of test video and Fig 8(b) is the result of subtracting the seventh frame from the reference or background frame.

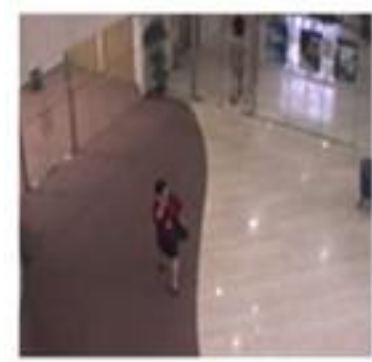

(a)

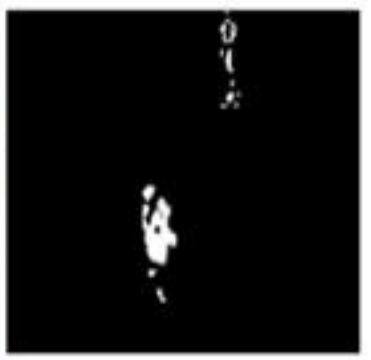

(b)
Fig 8: (a) Seventh Frame of Video (b) Background Subtraction 


\subsection{Results of Object Tracking}

The process of Tracking depends on the result of the frames of background subtraction. Proposed algorithm of tracking

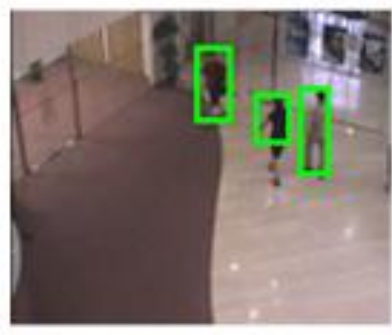

(a)

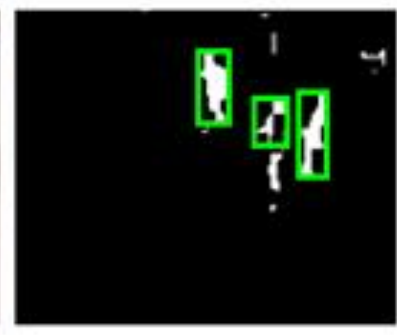

(b)
Fig 9: (a) Tracking of first frame (b) Tracking of first frame of background subtracted Video

can also be applied to the original frames of the video.

Fig 9 (a) is the result of object tracking in original frame of video. Fig 9(b) is the output of tracking of background subtracted frame. This background subtracted frame is the result obtained from the process of background subtraction.

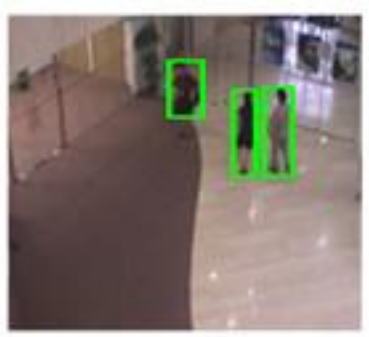

(a)

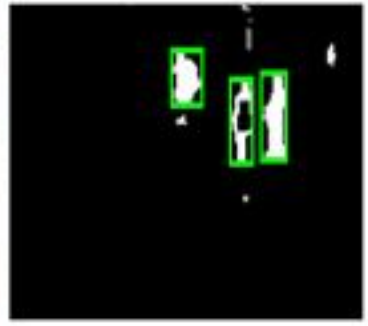

(b)
Fig 10: (a) Tracking of first frame (b) Tracking of first frame of background subtracted Video

Fig 10(a) is the result of object tracking in another frame of the video. Fig 10(b) is the output of tracking of background subtracted frame. This background subtracted frame is the result obtained from the process of background subtraction.

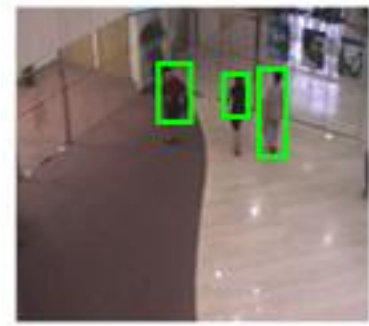

(a)

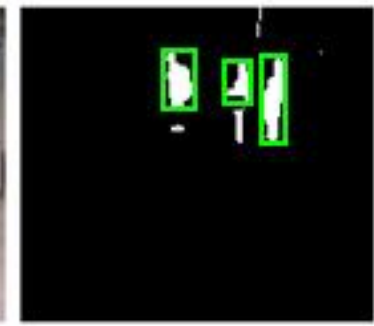

(b)
Fig 11: (a) Tracking of first frame (b) Tracking of first frame of background subtracted Video

Fig 11(a) is the result of tracking objects in the original frame of video. Fig 11(b) is the output of tracking of background subtracted frame. This background subtracted frame is the result obtained from the process of background subtraction

\section{DISCUSSION}

This algorithm has been tested on different type of images having different resolutions. The estimated time taken for different videos is calculated. Following is the performance of proposed algorithm.

Table 1: Comparison and results of different type of images

\begin{tabular}{|c|c|c|c|c|c|}
\hline $\begin{array}{l}\text { Image } \\
\text { Type }\end{array}$ & $\begin{array}{l}\text { Total } \\
\text { Frames }\end{array}$ & Resolution & $\begin{array}{l}\text { Processing } \\
\text { Time }\end{array}$ & $\begin{array}{l}\text { Maximu } \\
\mathrm{m} \text { No. of } \\
\text { objects }\end{array}$ & Results \\
\hline .bmp & 12 & $176 \times 144$ & $15.5229 \mathrm{sec}$ & 7 & $99 \%$ \\
\hline .bmp & 6 & $176 \times 144$ & $10.9178 \mathrm{sec}$ & 3 & $99 \%$ \\
\hline .bmp & 11 & $320 \times 156$ & $63.0647 \mathrm{sec}$ & 6 & $75 \%$ \\
\hline .jpg & 11 & $160 \times 120$ & $13.2904 \mathrm{sec}$ & 5 & $33 \%$ \\
\hline.$b m p$ & 11 & $160 \times 130$ & $14.2415 \mathrm{sec}$ & 3 & $30 \%$ \\
\hline
\end{tabular}

As shown in Table 1, the results of images of resolution $176 \times 144$ are much better than other images. On this resolution, proposed algorithm has been tested for 2 different number of frames i.e. 12 and 6 . Even though the processing time for 6 frames is smaller than the processing time of 12 frames but both gave the accuracy of $79 \%$ showing that increasing number of frames does not affect the overall system accuracy.

Result of images of resolution $320 \times 156$ is also somewhat satisfactory where increase in processing time is mainly due to increase in resolution. Accuracy of $75 \%$ is achieved.

The results of images of resolution 160x120 are not good enough but can be improved by changing the value of threshold.

Proposed algorithm has also been tested for the images of higher resolution such as $750 \times 526$ and got almost $0 \%$ results. This means that proposed algorithm cannot work for images of very high resolutions.

\section{CONCLUSION}

This paper discussed a unique and improved algorithm for detection and tracking of moving objects. The approach is based on a new technique for identifying the foreground pixels. It detects the foreground object completely and also it eliminates the shadows of that objects Experiments have been performed and this algorithm achieves detection of foreground objects to be more precise for the simple scenario and is observed that it eliminates the shadow of the moving objects. For the relatively complex background the postprocessing and filtering helps in improving the efficiency of the algorithm. Finally proposed algorithm is tested with a variety of images having various backgrounds and lighting conditions.

The algorithm for two-scan labeling which is capable of handling equivalences has been described here. This approach handles equivalences by means of class-merging steps. This approach can be found in the general labeling algorithm and is however different from the classical two-scan algorithm. The main merits are its simplicity and efficiency. The efficiency of proposed algorithms are shown by examples and results of the experiments conducted. This approach differs from the classical approach as it does not handle un-needed 
equivalences. In short this algorithm is remarkably simple to understand and to implement.

\section{REFERENCES}

[1] DeepaKumari, ShamikTiwari, Deepika Gupta, Raina , "Analysis on Adaptive Moving Objects via Robot Vision Implementations by Detection Techniques", International Journal of Scientific \& Engineering Research, Volume 3, Issue 4, April2012.

[2] ChandrashekharD.Badgujar, DipaliP.Sapkal, "A Survey on Object Detect, Track and Identify Using Video Surveillance", IOSR Journal of Engineering (IOSRJEN), Volume 2, Issue 10 (October 2012), PP 71-76.

[3] W.L. Khong, W.Y. Kow, H.T. Tan, H.P. Yoong, K.T.K. Teo, "Kalman Filtering Based Object Tracking in Surveillance Video System", Proceedings of the 3rd (2011) CUTSE International Conference, Miri, Sarawak, Malaysia, 8-9 Nov, 2011.

[4] Chirag I. Patel and Ripal Patel, "Illumination Invariant Moving Object Detection", International Journal of Computer and Electrical Engineering, Vol. 5, No. 1, February 2013.

[5] Shireen Y. Elhabian, Khaled M. El-Sayed and Sumaya H. Ahmed, "Moving Object Detection in Spatial Domain using Background Removal Techniques - State-of-Art", Recent Patents on Computer Science 2008, 1, 32-54.

[6] U.Chandrasekhar, Tapankumar Das, "A Survey of Techniques for Background Subtraction and Traffic Analysis on Surveillance Video", ISSN, Tapankumar das et al, UNIASCIT, Vol 1 (3), 2011, 107-113.
[7] Bo yang, Chang Huang, Ram Nevatia, "Segmentation of objects in a detection window by Nonparametric Inhomogeneous CRFs", Computer Vision and Image Understanding, Volume 115 Issue 11, November, 2011, Pages 1473-1482.

[8] Ritika, Gianetan Singh Sekhon, "Moving Object Analysis Techniques In Videos - A Review",IOSR Journal of Computer Engineering (IOSRJCE) ISSN, 2278-0661 Volume 1, Issue 2 (May-June 2012), PP 07-12

[9] A.Yilmaz, O. Javed, and M. Shah, "Object tracking: A survey",ACM Computing Surveys, Vol. 38, No. 4, 1-45, 2006

[10] Lucchese L., Mitra S. K. Color image segmentation A state-of-the-art survey. in Proc. Indian National Science Academy(INSA-A), vol. 67, A, New Delhi, India, Mar. 2001, pp. 207-221.

[11] Stauffer C, Grimson W.E.L. Adaptive background mixture models for real-time tracking. Proceedings of IEEE Conference on Computer Vision and Pattern Recognition. Ft. Collins, 1999: 246-252

[12] Power P. W., Schoonees J. A. Understanding background mixture models for foreground segmentation. In Proceedings Image and Vision Computing, 2002, pp:267-271

[13] Lee D.S., Hull J., Erol B. A Bayesian framework for gaussian mixture background modeling. in Proceedings of IEEE International Confererence on Image Processing, 2003, pages:973-976

[14] S. Gundimada, Li Tao, and v. Asari, "Face detection technique based on intensity and skin color distribution," in 2004 International Conference on Image Processing, Otc. 2004, vol. 2, pp. 1413-1416.

[15] K. P. Seng, A. Suwandy, and L.-M. Ang, "Improved automatic face detection technique in color images," in IEEE Region 10 Conference TENCON 2004, Nov. 2004, vol. 1,pp. 459-462. 